\title{
Original
}

\section{Dolor Lumbar en pacientes de consulta traumatológica. Variables socio-sanitarias relacionadas}

\section{Low Back Pain in Trauma Patients. Related Social and Health Variables}

\author{
$M^{a}$ Teófila Vicente-Herrero', Servio Tulio Casal-Fuentes², Gemma Victoria Espí-López³, Alejandro Fernández- \\ Montero ${ }^{4}$
}

1. Servicio de Prevención de Riesgos Laborales. Área de Medicina del Trabajo. Grupo Correos. Valencia. España.

2. Servicio de Prevención de Riesgos Laborales ASPY Prevención. Valencia. España.

3. Departamento de Fisioterapia. Universidad de Valencia. Valencia. España.

4. Servicio de Prevención de Riesgos Laborales, Área de Medicina del Trabajo, Universidad de Navarra, Pamplona. España.

\section{Recibido: 11-06-2019}

Aceptado: 21-08-2019

\section{Correspondencia}

Ma Teófila Vicente-Herrero

Servicio Medicina del trabajo-Grupo Correos

Plaza del ayuntamiento 24-2

46002 Valencia. España

Correo electrónico: mtvh@ono.com

\section{Resumen}

Introducción: El dolor lumbar es patología prevalente influida por factores ambientales y personales: edad, sexo, nivel educativo, estrés, apoyo social y riesgos laborales, entre otros.

Es objetivo de este trabajo es valorar la repercusión de variables sociodemográficas en trabajadores con lumbalgia así como su impacto en discapacidad.

Material y Método: Estudio descriptivo en 351 pacientes diagnosticados de dolor lumbar. Se valora la influencia de: edad, sexo, hábitos de vida y limitaciones con el test Oswestry.

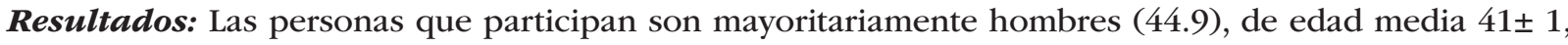
y un nivel de estudios elemental (47.4). Los hábitos de vida más saludables se dan entre la población joven (81.4\%) y en las mujeres (82.6\%), el consumo de tabaco aumenta con la edad (en $>50$ años el $55 \%$ es fumador) y las mujeres toman menos alcohol ( $40.1 \%$ no consume), pero practican menos ejercicio físico (diario el 6.6\%). En jóvenes es más efectiva la fisioterapia (57.7\%), mientras que los mayores combinan fármacos y fisioterapia (45\%).

La repercusión de lumbalgia en incapacidad aumenta con la edad, especialmente en mujeres y mantiene una relación positiva con el grado de discapacidad del Oswestry $(\mathrm{p}<0.005)$. Limita la bipedestación y dificulta dormir variando los resultados con la edad. En las mujeres destacan las limitaciones para actividades cotidianas, deambulación y actividades sociales $(\mathrm{p}<0.005)$.

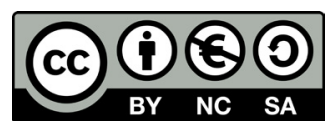

https://creativecommons.org/licenses/by-nc-sa/4.0/ 
Conclusiones: Las variables sociodemográficas y culturales influyen en el dolor lumbar, especialmente edad, sexo y hábitos de vida. El test de Oswestry utilizado correlaciona las limitaciones por lumbalgia con la repercusión en incapacidad laboral.

Med Segur Trab (Internet). 2019;65(256):186-198

Palabras clave: dolor lumbar, edad, sexo, test Oswestry, salud laboral

\section{Abstract}

Introduction: Low back pain is a prevalent pathology influenced by environmental and personal factors: age, gender, educational level, stress, social support and occupational risks, among others.

The aim of this work is to assess the impact of socio-demographic variables of workers with low back pain and its disability impact.

Material and metbod: Descriptive study of 351 low-back pain patients. The influence of gender, sex, life habits and limitations with the Oswestry questionnaire is assessed.

Results: The participants, mainly men and an average age of $41 \pm 1$ years have an primary education. Youth population $(81.4 \%)$ and women $(82.6 \%)$ have the healthiest habits, tobacco consumption increases with age (> 50 years, 55\%) and women drink less alcohol (40.1\% don't consume) but practice less physical exercise (daily 6.6\%). Physiotherapy is more effective for youth people $(57.7 \%)$, while elderly people combine drugs and physiotherapy (45\%).

The impact of low back pain on disability increases with age, especially in women, keeping a positive relationship with the Oswestry Disability Questionnaire index ( $\mathrm{p}<0.005)$. It limits the ability to stand and makes sleeping difficult, varying the results along age. In women, the limitations for daily activities, walking and social activities stand out $(\mathrm{p}<0,005)$.

Conclusions: Socio-demographic and cultural variables, specially age, gender and lifestyle habits, have an influence on low-back pain. The Oswestry Questionnaire used correlates the limitations for low back pain with the repercussion in work disability.

Med Segur Trab (Internet). 2019;65(256):186-198

Keywords: low back pain, age, gender, Oswestry Questionnaire, occupational health 


\section{INTRODUCCIÓN}

El dolor lumbar es un problema común que la mayoría de las personas experimenta en algún momento de su vida. Aunque en todos los estudios se destaca su alta prevalencia, la heterogeneidad entre ellos dificulta poder realizar comparativas y agrupar datos. Las estimaciones sobre la incidencia de un primer episodio de dolor de lumbalgia oscilan entre el $6,3 \%$ y el $15,4 \%$, y la mayoría de las personas que experimentan dolor lumbar refieren limitaciones en su actividad y episodios recurrentes que van del $24 \%$ al $80 \%$, supone el $4,39 \%$ de las urgencias hospitalarias ${ }^{1}$. Muchos factores ambientales y personales influyen en el inicio y el curso del dolor lumbar, destacando la edad, bajo nivel educativo, estrés, ansiedad, depresión, insatisfacción laboral, bajo apoyo social en el lugar de trabajo y riesgos laborales como las vibraciones en todo el cuerpo ${ }^{2}$. La combinación de factores de riesgo psicológicos, sociales, genéticos, junto con los mecanismos cerebrales implicados en el dolor lumbar ponen de relieve la necesidad de un enfoque multidisciplinario integrando aspectos biológicos, psicológicos y sociales ${ }^{3}$.

En todos los países se apoya la idea de incrementar los estudios de investigación que aporten datos más amplios acerca del dolor lumbar ya que, a pesar de los avances en técnicas diagnósticas y terapéuticas, sigue siendo un padecimiento extremadamente común. La educación del paciente es factor prioritario para evitar el riesgo de reincidencias posteriores con el objetivo concreto de aliviar tanto el dolor, como la capacidad funcional ${ }^{4}$.

La prevalencia de esta patología en población general y, especialmente en personas que trabajan, asociada a variables personales, sociales y laborales orienta a realizar estudios que apoyen enfoques preventivos, diagnósticos y terapéuticos para detectar aquellas características que permitan mejorar su curso evolutivo y prevenir riesgos laborales.

\section{OBJETIVO}

Valorar las variables (edad, sexo, hábitos de vida y consumos de tabaco y alcohol) recogidas de la historia clínica de pacientes con lumbalgia procedentes de una unidad de traumatología de la comunidad valenciana en España y su repercusión en las limitaciones medidas con la escala analógica de dolor y con el cuestionario de Oswestry en incapacidad laboral.

\section{MATERIAL Y MÉTODO}

Estudio descriptivo en el que participan 351 sujetos procedentes de consulta de Unidad de Traumatología de la Malvarrosa en Valencia (España). Son criterios de inclusión padecer de lumbalgia y estar en activo laboralmente (se excluyen desempleados), no superar los 65 años (edad laboral), participación voluntaria, consentimiento escrito de autorización y uso epidemiológico de los datos y autorización del comité ético de la entidad sanitaria para la realización del estudio.

Todos los procedimientos se realizaron de acuerdo con los principios de la Declaración de Helsinki de la Asociación Médica Mundial y los protocolos fueron aprobados por el Comité de Ética en Investigación Humana del Hospital Clínico de Valencia (F-CE-GEva-15-v1.1 de 23 de julio de 2015).

Se realizó una entrevista clínica que incluyó datos sociodemográficos como edad, sexo, hábitos de vida, consumos de tabaco y alcohol, dolor y limitación funcional.

La edad de los participantes fue categorizada en tres grupos: $<40$ años, 40-49 años y $\geq 50$ años. El consumo de tabaco se categoriza en: nunca fumador, fumador actual y exfumador (al menos 1 año sin consumo de tabaco) y el cálculo paquetes/día según el 
número de cigarrillos que fuma al día multiplicado por número de años que lleva fumando, y dividido entre 20 (uso de cálculo electrónico)(1). Se considera alimentación saludable, a criterio de los autores, la toma de frutas y verduras al menos 3 veces/semana. La práctica de ejercicio físico se categoriza a criterio de los autores en: nunca, ocasional, 1-3/días por semana, 3-6 días por semana y diario. El consumo de alcohol se categoriza partiendo de los gramos de alcohol consumido (calculo UBE) 5 : no consumo, consumo de bajo riesgo-leve (< de $11 \mathrm{UBE}$ por semana en mujeres y menos de 17 en hombres), consumo de riesgo moderado (entre 11 y 16 en mujeres y 17-27 en hombres) y consumo severo- abusivo ( $>$ de 17 en mujeres y de 28 en hombres).

La intensidad del dolor se mide con escala analógica digital siendo categorizada por los autores para un mejor manejo en: Ausencia de dolor-Dolor leve si puntúa $\leq 6$, Dolor moderado si puntúa entre 7-8 y Dolor severo si puntúa $\geq 9^{6}$.

El impacto en limitaciones se evaluó atendiendo a la escala-cuestionario de incapacidad por dolor lumbar de Oswestry, en su adaptación transcultural a la población española de Flórez García MT at al en 1995․ La escala tiene 10 cuestiones con 6 posibles respuestas cada una y se valoran individualmente cada una, además de la valoración global. Cada ítem se valora de 0 a 5, de menor a mayor limitación. Puntuación: el test consta de 10 ítems, cada ítem consta de 6 opciones que se puntúan de 0-5. La suma total máxima de puntuación de todos los ítems es de 50 y el porcentaje de limitación/ discapacidad resulta de dividir la puntuación obtenida por 50 y multiplicar el resultado por 100. Clasificación: 0-20 \% limitación funcional mínima, 20 \%-40 \% limitación funcional moderada, 40 \%-60 \% limitación funcional intensa, 60 \%-80\% discapacidad, por encima de 80 \% limitación funcional máxima (Anexo). El cuestionario presenta unas características métricas adecuadas ${ }^{8}$.

El cuestionario de Oswestry es el que se ha utilizado en este trabajo y actualmente es uno de los más utilizados para medir la discapacidad en los trastornos de la columna vertebral. En 1995 se tradujo y se adaptó de manera transcultural al contexto español. Su contenido y validez aparente, consistencia interna y confiabilidad test-retest se demostraron para una población española con dolor lumbar y se reafirma en sus buenas propiedades psicométricas (consistencia interna y validez de constructo), convirtiéndolo en un instrumento válido para la medición de la discapacidad en pacientes españoles, específicamente en pacientes con discapacidad moderada / intensa? .

Este Índice de discapacidad, del cual se han realizado 16 adaptaciones interculturales, es una medida de resultado basada en autoinforme que se utiliza para cuantificar el grado de discapacidad relacionada con el dolor lumbar, un factor importante que contribuye al ausentismo y absentismo en el lugar de trabajo, aspecto de importancia laboral si consideramos que es la segunda causa de discapacidad en el mundo, solo detrás del cáncer $^{10}$. Junto con el Cuestionario de Discapacidad Roland-Morris, el Índice de Discapacidad Oswestry es el más utilizado para valorar discapacidad y repercusión en absentismo laboral por lumbalgia. Según los estudios comparativos directos existentes, no hay razones sólidas para preferir uno de ellos y se necesitan estudios de mayor calidad para confirmar esta conclusión, evaluando y comparando la validez de contenido estructural y transcultural de estos cuestionarios en pacientes con dolor lumbar ${ }^{11}$.

\section{Análisis estadístico}

Para valorar las características de la población, los participantes se agruparon por sexo y edad. Se calcularon las frecuencias de las variables categóricas del cuestionario y medidas de tendencia central y desviación estándar en las cuantitativas. Para comparar los porcentajes entre grupos de las diferentes variables se utilizó el test Chi- cuadrado y el test de la t-de student para comparar medias.

(1) https://www.interna.es/calculadoras/calculadoras-escalas/paquetes-ano/ 
Para valorar la asociación entre el porcentaje de discapacidad lumbar (agrupado en terciles) y el riesgo de baja laboral por enfermedad se realizó una regresión logística ajustada por edad y sexo (OR IC95\%) Además se realizó una representación gráfica, para analizar la correlación entre la discapacidad lumbar y los días de baja por enfermedad.

El nivel de significación estadística en todos los casos fue de $P<0.05$. El análisis estadístico se llevó a cabo mediante el programa STATA versión 12.0.

\section{RESULTADOS}

Participan 349 sujetos, de los que 197 son hombres (56.4\%) y 152 mujeres (43,6\%) con una edad media de 44 años (44,9 los hombres y 43 las mujeres) y un nivel de estudios elemental en el 47,4\% de la población global, 31,5 con estudios de grado medio y 21,1 de grado superior. En las mujeres es algo mayor el porcentaje de trabajadoras con estudios superiores (21,5\% frente al $20,8 \%$ de los hombres) y también tienen un porcentaje algo mayor de estudios de grado medio (34,9\%, frente al $28,9 \%$ de los hombres), en los hombres es mayor el porcentaje con estudios elementales $(50,3 \%$ frente al 43,6\% de las mujeres).

Las diferencias que se encuentran en la población participante atendiendo al grupo de edad se muestran en las tablas I y II.

Tabla I. Relación de la edad con variables personales.

\begin{tabular}{|c|c|c|c|c|c|}
\hline & Edad & $<40$ & $40-49$ & $\geq 50$ & $\mathbf{p}$ \\
\hline \multirow{4}{*}{ Consumo de tabaco } & No fumador & 55.7 & 36.6 & 25 & \multirow{3}{*}{0.001} \\
\hline & Exfumador & 12.4 & 15.7 & 20 & \\
\hline & Fumador & 32 & 47.7 & 55 & \\
\hline & Paquetes año & $2.27(3.76)$ & $8.69(10.48)$ & $16.98(14.74)$ & $<0.001$ \\
\hline Sexo (referencia mujer) & & 53.6 & 43.6 & 31.3 & 0.012 \\
\hline Alimentación saludable & $\begin{array}{l}\text { Consumo de fruta/ } \\
\text { verdura }\end{array}$ & 81.4 & 59.2 & 35 & $<0.001$ \\
\hline \multirow{5}{*}{ Ejercicio físico } & Nunca/Casi nunca & 2.1 & 20.3 & 46.3 & \multirow{5}{*}{$<0.001$} \\
\hline & Ocasional & 10.3 & 23.3 & 30 & \\
\hline & $1-3 \mathrm{~d} /$ semana & 66 & 41.9 & 23.7 & \\
\hline & 3-6 d/semana & 10.31 & 10.47 & 0 & \\
\hline & A diario & 11.34 & 4.07 & 0 & \\
\hline \multirow{4}{*}{ Consumo de alcohol } & No consume alcohol & 29.9 & 23.3 & 13.8 & \multirow{4}{*}{$<0.001$} \\
\hline & Leve & 60.8 & 55.8 & 26.2 & \\
\hline & Moderado & 8.2 & 14.5 & 37.5 & \\
\hline & Alto & 1.03 & 6.4 & 22.5 & \\
\hline \multirow{4}{*}{$\begin{array}{l}\text { Episodios de lumbalgia/ } \\
\text { último año }\end{array}$} & Ninguno & 9.28 & 8.72 & 3.75 & \multirow{4}{*}{$<0.001$} \\
\hline & Uno & 45.4 & 18.6 & 28.7 & \\
\hline & 1 a 3 & 40.2 & 57 & 43.8 & \\
\hline & Más de 3 & 5.2 & 15.7 & 23.7 & \\
\hline Antecedentes traumatoló & icos o reumatológicos & 3.1 & 30.2 & 22.1 & $<0.001$ \\
\hline \multirow{2}{*}{$\begin{array}{l}\text { Repercusión en } \\
\text { Incapacidad Temporal } \\
\text { (IT) }\end{array}$} & $\begin{array}{l}\text { Procesos de IT/baja } \\
\text { laboral (\%) }\end{array}$ & 5.4 & 31.7 & 24.7 & $<0.001$ \\
\hline & Días de IT/baja laboral & $\begin{array}{c}30 \\
(15)\end{array}$ & $153(118)$ & $\begin{array}{c}272 \\
(158)\end{array}$ & 0.002 \\
\hline
\end{tabular}


Tabla II. Relación de la edad con variables clínicas relacionadas con lumbalgia.

\begin{tabular}{|c|c|c|c|c|c|}
\hline & Edad & $<40$ & $40-49$ & $\geq 50$ & $\mathbf{p}$ \\
\hline \multirow{3}{*}{ Intensidad de dolor } & Leve & 30.9 & 32.6 & 42.5 & \multirow{3}{*}{0.001} \\
\hline & Moderado & 57.7 & 61 & 36.3 & \\
\hline & Intenso & 11.3 & 6.4 & 21.3 & \\
\hline \multirow{3}{*}{ Tratamiento recibido } & Fisioterapia & 57.7 & 37.8 & 45 & \multirow{3}{*}{0.019} \\
\hline & Farmacológico & 5.15 & 13.95 & 10 & \\
\hline & Ambos & 37.1 & 48.3 & 45 & \\
\hline \multirow{3}{*}{$\begin{array}{l}\text { Efectividad del tratamiento } \\
\text { recibido }\end{array}$} & Nada & 30.6 & 26.8 & 29.9 & \multirow{3}{*}{0,19} \\
\hline & Parcialmente & 67.1 & 73.2 & 70.1 & \\
\hline & Completamente & 2.35 & 0 & 0 & \\
\hline \multirow{3}{*}{$\begin{array}{l}\text { Limitaciones para realizar } \\
\text { actividades cotidianas }\end{array}$} & Ninguna & 89.7 & 89 & 81.3 & \multirow{3}{*}{0.33} \\
\hline & Leve & 9.3 & 9.9 & 15 & \\
\hline & Moderada & 1.03 & 1.16 & 3.75 & \\
\hline \multirow{3}{*}{$\begin{array}{l}\text { Limitaciones para } \\
\text { Manipulación de Cargas }\end{array}$} & Ninguna & 86.6 & 93 & 85 & \multirow{3}{*}{0.11} \\
\hline & Leve & 11.3 & 6.4 & 15 & \\
\hline & Moderada & 2.06 & .58 & 0 & \\
\hline \multirow{2}{*}{$\begin{array}{l}\text { Limitaciones para la } \\
\text { deambulación }\end{array}$} & Ninguna & 88.7 & 84.3 & 86.3 & \multirow{2}{*}{0.61} \\
\hline & Leve & 11.3 & 15.7 & 13.8 & \\
\hline \multirow{2}{*}{$\begin{array}{l}\text { Limitaciones para la } \\
\text { sedestación }\end{array}$} & Ninguna & 83.5 & 77.3 & 75 & \multirow{2}{*}{0.34} \\
\hline & Leve & 16.5 & 22.7 & 25 & \\
\hline \multirow{2}{*}{$\begin{array}{l}\text { Limitaciones para la } \\
\text { bipedestación }\end{array}$} & Ninguna & 82.5 & 82 & 68.8 & \multirow{2}{*}{0.035} \\
\hline & Leve & 17.5 & 18 & 31.3 & \\
\hline \multirow{4}{*}{$\begin{array}{l}\text { Limitaciones para dormir } \\
\text { por lumbalgia }\end{array}$} & Ninguna & 89.7 & 88.4 & 77.5 & \multirow{4}{*}{0.017} \\
\hline & Leve & 7.22 & 7.56 & 15 & \\
\hline & Moderada & 1.03 & 0 & 5 & \\
\hline & Grave & 2.06 & 4.07 & 2.5 & \\
\hline \multirow{2}{*}{$\begin{array}{l}\text { Limitaciones en relaciones } \\
\text { sexuales }\end{array}$} & Ninguna & 92.8 & 97.1 & 88.7 & \multirow{2}{*}{0.29} \\
\hline & Leve & 7.22 & 2.91 & 11.25 & \\
\hline \multirow{3}{*}{$\begin{array}{l}\text { Limitaciones para } \\
\text { actividades sociales }\end{array}$} & Ninguna & 93.8 & 90.7 & 85 & \multirow{3}{*}{0.38} \\
\hline & Leve & 5.15 & 8.14 & 12.5 & \\
\hline & Moderada & 1.03 & 1.16 & 2.5 & \\
\hline \multirow{2}{*}{ Limitaciones para viajar } & Ninguna & 78.4 & 79.7 & 71.2 & \multirow{2}{*}{0.32} \\
\hline & Leve & 21.6 & 20.3 & 28.7 & \\
\hline
\end{tabular}

En el colectivo de personas que participan en el estudio el consumo de tabaco aumenta con la edad ( $\mathrm{p} 0,001)$, al igual que la cantidad de tabaco consumida en paquetes/ año $(\mathrm{p}<0,001)$. Los sujetos más jóvenes tienen hábitos de alimentación más saludables (con mayor consumo de frutas y verduras) $(\mathrm{p}<0,001)$ y mayor práctica de ejercicio físico $(\mathrm{p}<0,001)$. El consumo de alcohol moderado-alto aumenta con la edad, siendo nulo o leve en la población más joven $(\mathrm{p}<0,001)$.

Los episodios de lumbalgia aumentan con la edad y cuando se dan en jóvenes son menos de 3 por año $(\mathrm{p}<0,001)$. A partir de los 40 años aumentan los antecedentes traumatológicos o reumatológicos $(\mathrm{p}<0,001)$ y también aumenta la repercusión en incapacidad laboral/procesos de baja laboral con la edad $(\mathrm{p}<0,001)$ y lo mismo ocurre con la duración de las bajas laborales aunque sin significación estadística en los resultados obtenidos. 
En las personas más jóvenes el dolor por lumbalgia es leve o moderado, mientras que en los más mayores es más intenso (p 0,001). La fisioterapia es más efectiva en personas jóvenes, mientras que en los mayores se requiere del uso combinado de fármacos y fisioterapia (p 0,019).

La lumbalgia implica limitaciones para la bipedestación y para dormir con intensidad variable según los distintos grupos de edad y sin que se pueda establecer una significación estadística en ninguno de los ítems valorados.

Las diferencias que se encuentran en la población participante atendiendo al sexo se muestran en las tablas III y IV.

Tabla III. Relación del sexo con variables personales.

\begin{tabular}{|c|c|c|c|c|}
\hline & Sexo & Hombre & Mujer & $\mathbf{p}$ \\
\hline \multirow{4}{*}{ Consumo de tabaco } & No fumador & 27.9 & 53.9 & \multirow{3}{*}{$<0.001$} \\
\hline & Exfumador & 19.3 & 11.2 & \\
\hline & Fumador & 52.8 & 34.9 & \\
\hline & Paquetes año & $\begin{array}{c}11.04 \\
(12.16)\end{array}$ & $\begin{array}{c}5.7 \\
(9.86)\end{array}$ & $<0.001$ \\
\hline edad & & $44.9(6.7)$ & $\begin{array}{l}43 \\
(7)\end{array}$ & 0.01 \\
\hline Alimentación saludable & Consumo de fruta/verdura & 42.6 & 82.6 & $<0.001$ \\
\hline \multirow{5}{*}{ Ejercicio físico } & Nunca/Casi nunca & 26.9 & 13.8 & \multirow{5}{*}{0.005} \\
\hline & Ocasional & 20.8 & 21.7 & \\
\hline & $1-3 \mathrm{~d} /$ semana & 43.7 & 45.4 & \\
\hline & 3-6 d/semana & 4.57 & 12.5 & \\
\hline & A diario & 4.06 & 6.58 & \\
\hline \multirow{4}{*}{ Consumo de alcohol } & No consume alcohol & 9.6 & 40.1 & \multirow{4}{*}{$<0.001$} \\
\hline & Leve & 48.7 & 52.6 & \\
\hline & Moderado & 26.4 & 7.2 & \\
\hline & Alto & 15.23 & 0 & \\
\hline \multirow{4}{*}{$\begin{array}{l}\text { Episodios de } \\
\text { lumbalgia/último año }\end{array}$} & Ninguno & 7.61 & 7.89 & \multirow{4}{*}{0.495} \\
\hline & Uno & 31.5 & 24.3 & \\
\hline & 1 a 3 & 47.7 & 51.3 & \\
\hline & Más de 3 & 13.2 & 16.4 & \\
\hline \multicolumn{2}{|c|}{ Antecedentes traumatológicos o reumatológicos } & $125(119)$ & $\begin{array}{l}196 \\
(144)\end{array}$ & 0.046 \\
\hline \multirow{2}{*}{$\begin{array}{l}\text { Repercusión en } \\
\text { Incapacidad Temporal } \\
\text { (IT) }\end{array}$} & $\begin{array}{l}\text { Procesos de IT/baja } \\
\text { laboral (\%) }\end{array}$ & 14.9 & 28.2 & 0.003 \\
\hline & Días de IT/baja laboral & 11.6 & 37.9 & $<0.001$ \\
\hline
\end{tabular}

El consumo de tabaco es mayor en el colectivo de hombres y también la cantidad de tabaco consumida $(\mathrm{p}<0,001)$. Las mujeres llevan una alimentación más saludable $(\mathrm{p}<0,001)$ pero practican menos ejercicio físico que los hombres ( $\mathrm{p} 0,005$ ) y consumen menos alcohol, cuando lo hacen es en cantidad moderada $(\mathrm{p}<0,001)$.

Las mujeres presentan en mayor número antecedentes de patologías traumatológicas o reumatológicas, si bien los resultados no son significativos, y tienen mayor repercusión en procesos de IT y duración de los mismos por lumbalgia que los hombres $(\mathrm{p}<0,001)$.

Se observa una relación positiva creciente entre el grado de discapacidad cuantificado en porcentaje de limitación del test Oswestry y la repercusión en IT de la patología valorada en días de baja por la enfermedad $(p<0,05)$ (figura 1). 
Tabla IV. Relación del sexo con variables clínicas relacionadas con lumbalgia

\begin{tabular}{|c|c|c|c|c|}
\hline & Sexo & Hombre & Mujer & $\mathbf{p}$ \\
\hline \multirow{3}{*}{ Intensidad de dolor } & Leve & 39.6 & 27.6 & \multirow{3}{*}{0.06} \\
\hline & Moderado & 50.8 & 59.2 & \\
\hline & Intenso & 9.6 & 13.2 & \\
\hline \multirow{3}{*}{ Tratamiento recibido } & Fisioterapia & 47.7 & 41.4 & \multirow{3}{*}{0.08} \\
\hline & Farmacológico & 12.7 & 7.9 & \\
\hline & Ambos & 39.6 & 50.7 & \\
\hline \multirow{3}{*}{$\begin{array}{l}\text { Efectividad del tratamiento } \\
\text { recibido }\end{array}$} & Nada & 28.3 & 28.7 & \multirow{3}{*}{0.29} \\
\hline & Parcialmente & 71.7 & 69.9 & \\
\hline & Completamente & 0 & 1.4 & \\
\hline \multirow{3}{*}{$\begin{array}{l}\text { Limitaciones para realizar } \\
\text { actividades cotidianas }\end{array}$} & Ninguna & 91.4 & 82.2 & \multirow{3}{*}{0.037} \\
\hline & Leve & 7.6 & 15.1 & \\
\hline & Moderada & 1.02 & 2.63 & \\
\hline \multirow{3}{*}{$\begin{array}{l}\text { Limitaciones para } \\
\text { Manipulación de Cargas }\end{array}$} & Ninguna & 91.9 & 86.2 & \multirow{3}{*}{0.22} \\
\hline & Leve & 7.6 & 12.5 & \\
\hline & Moderada & .51 & 1.32 & \\
\hline \multirow{2}{*}{$\begin{array}{l}\text { Limitaciones para la } \\
\text { deambulación }\end{array}$} & Ninguna & 89.3 & 81.6 & \multirow{2}{*}{0.039} \\
\hline & Leve & 10.7 & 18.4 & \\
\hline \multirow{2}{*}{$\begin{array}{l}\text { Limitaciones para la } \\
\text { sedestación }\end{array}$} & Ninguna & 78.2 & 78.9 & \multirow{2}{*}{0.86} \\
\hline & Leve & 21.8 & 21.1 & \\
\hline \multirow{2}{*}{$\begin{array}{l}\text { Limitaciones para la } \\
\text { bipedestación }\end{array}$} & Ninguna & 82.2 & 75 & \multirow{2}{*}{0.10} \\
\hline & Leve & 17.8 & 25 & \\
\hline \multirow{4}{*}{$\begin{array}{l}\text { Limitaciones para dormir } \\
\text { por lumbalgia }\end{array}$} & Ninguna & 85.3 & 87.5 & \multirow{4}{*}{0.22} \\
\hline & Leve & 10.15 & 7.89 & \\
\hline & Moderada & .51 & 2.63 & \\
\hline & Grave & 4.06 & 1.97 & \\
\hline \multirow{2}{*}{$\begin{array}{l}\text { Limitaciones en relaciones } \\
\text { sexuales }\end{array}$} & Ninguna & 94.4 & 93.4 & \multirow{2}{*}{0.70} \\
\hline & Leve & 5.58 & 6.58 & \\
\hline \multirow{3}{*}{$\begin{array}{l}\text { Limitaciones para } \\
\text { actividades sociales }\end{array}$} & Ninguna & 93.4 & 86.2 & \multirow{3}{*}{0.045} \\
\hline & Leve & 5.08 & 12.5 & \\
\hline & Moderada & 1.52 & 1.32 & \\
\hline \multirow{2}{*}{ Limitaciones para viajar } & Ninguna & 77.7 & 77 & \multirow{2}{*}{0.88} \\
\hline & Leve & 22.3 & 23 & \\
\hline
\end{tabular}

En las mujeres la repercusión de la lumbalgia para realizar las actividades de la vida cotidiana es mayor, aunque de forma leve o moderada. Es también mayor la limitación en deambulación y para actividades sociales $\mathrm{p}<0,05$ )

\section{DISCUSIÓN}

El uso de cuestionarios para valorar y cuantificar la capacidad funcional de pacientes con dolor lumbar es habitual, Son varios los cuestionarios utilizados, cada cual con sus ventajas y limitaciones. Los más utilizados son: Low Back Pain Rating Scale (LBPRS), Oswestry Disability Index (ODI), Progressive Isoinertial LiftingEvaluation (PILE), Quebec Back Pain Disability Scale (QBPDS), and Roland-Morris Disability Questionnaire (RDQ) ${ }^{12}$. A pesar de ello, existe una necesidad insatisfecha de un instrumento validado que permita 
evaluar los síntomas e impactos relacionados con el dolor lumbar para su uso en ensayos y en la clínica habitual ${ }^{13}$.

Figura 1. Correlación entre discapacidad lumbar medida mediante el test de Oswestry y días de baja por enfermedad laboral.

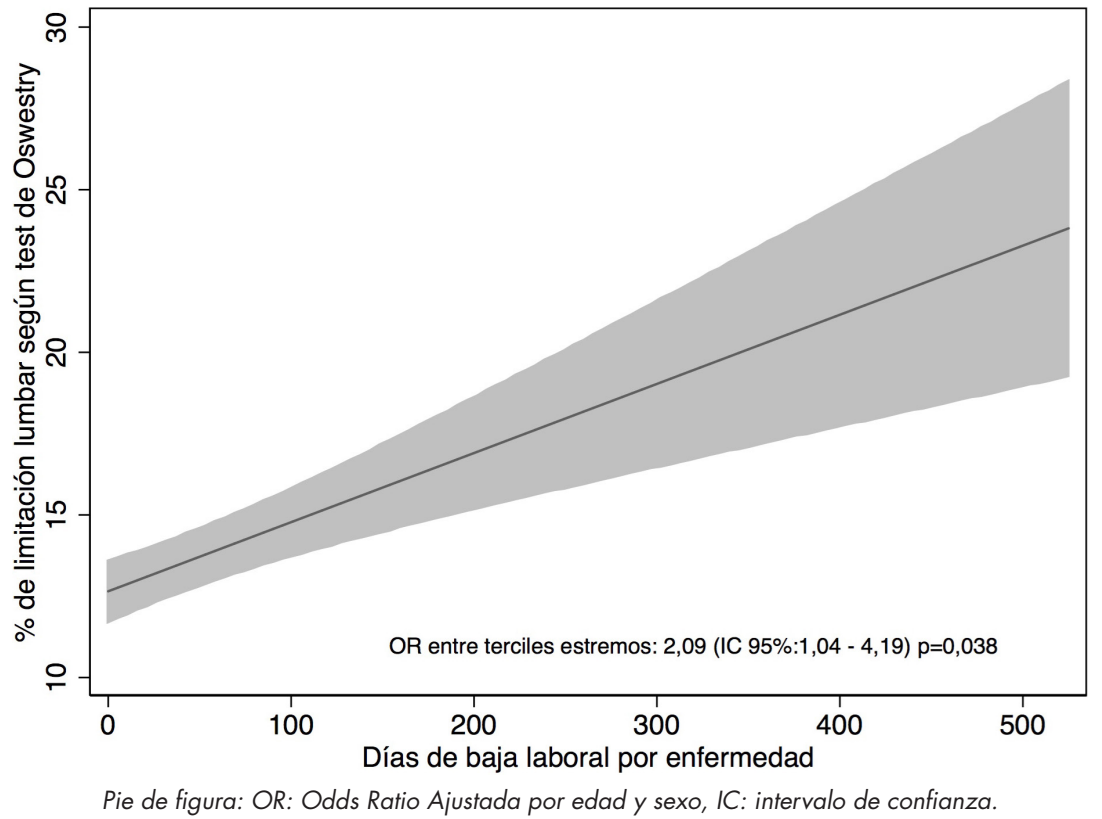

Uno de los más usados es el cuestionario de Owestry ${ }^{14}$, que es también el que se ha utilizado en nuestro estudio, donde los resultados muestran la influencia de variables como la edad y el sexo en el dolor lumbar, siendo coincidentes con lo aportado por estudios previos, como el realizado por La Asociación Ortopédica Japonesa, que mediante el uso del Cuestionario de Evaluación del Dolor en la Espalda de JOA (JOABPEQ) ${ }^{15}$ evalúa aspectos del dolor lumbar en pacientes que guardan relación con la edad, aunque en este trabajo la edad de los participantes era algo mayor $(56,1)$ que la de nuestro estudio. En cuanto al sexo obtuvo peor puntuación en la escala analógica de dolor lumbar en los hombres que en las mujeres, al contrario de lo obtenido en nuestro estudio donde parece observarse una tendencia a dolor moderado/intenso en las mujeres en mayor proporción que en los hombres, aunque no observamos una significación estadística que permita concluir en esta afirmación. Según el tipo de enfermedad causal destaca la repercusión funcional en casos de hernia de disco o con estenosis espinal, aspecto este no valorado en nuestro trabajo. La repercusión en calidad de vida en nuestro caso se ha hecho con un cuestionario diferente, el Oswestry, aunque los resultados coinciden en mostrar una mayor afectación en deambulación y limitaciones sociales.

Otros estudios han valorado también el impacto de la edad y del factor género en la clasificación internacional de la evaluación basada en el funcionamiento, la discapacidad y la salud (ICF) para el dolor lumbar crónico, mostrando que ambos factores, la edad y el género influyen en la clasificación integral de los conjuntos básicos de ICF para el dolor crónico de espalda. Este impacto es relevante para las evaluaciones basadas en la ICF en la práctica clínica, y debe considerarse en la planificación de cualquier intervención en programas de rehabilitación y para las planificaciones de intervenciones basadas en el ICF ${ }^{16}$.

Estudios similares al nuestro se han realizado en países mediterráneos (Grecia) con estilos de vida muy parecidos y estimando la prevalencia de dolor lumbar y su asociación con factores sociodemográficos, físicos y de estilo de vida, en este caso, con el apoyo de la escala de ansiedad y depresión (HAD), SF-12 para la calidad de vida (QoL) y Roland- 
Morris para la discapacidad. Los resultados de este trabajo muestran algunas diferencias con los obtenidos en nuestro estudio, ya que las características sociodemográficas no se asociaron con factores físicos de dolor lumbar, excepto la edad que se correlacionó con la discapacidad física y el bienestar. Los factores físicos (particularmente la intensidad del dolor y la ubicación) se correlacionaron con los factores del estilo de vida (QoL) y con la discapacidad. Es por esto que los autores de este trabajo abren la opción de que haya un trasfondo socioeconómico y un dominio etiológico diferentes a los del espectro de dolor lumbar no específico habitual que justifique el hecho de que en su estudio la mayoría de las características sociodemográficas no se correlacionen con ningún factor físico o de estilo de vida en este tipo de dolor de localización lumbar ${ }^{17}$.

Sin embargo, la influencia de la edad y del sexo de los pacientes en la presencia de dolor lumbar es clara y, en algunos trabajos de revisión biomecánica, se destaca la repercusión en lordosis lumbar y en rango de movimiento de ambas variables, ya que son éstas medidas fisiológicas importantes al iniciar cualquier diagnóstico o plan de tratamiento en pacientes con lumbalgia, y muestran variaciones con diferencias significativas según edad y sexo. Las mujeres jóvenes mostraron una lordosis lumbar y rango de extensión significativamente mayor, mientras que los hombres jóvenes mostraron un rango de flexión mayor. Las diferencias de flexión lateral también mostraron diferencia significativa entre los diferentes grupos de edad en ambos sexos, por ello los autores destacan la importancia de distinguir entre los déficits funcionales causados por trastornos de la columna vertebral y factores / afecciones naturales relacionados con la edad y el sexo de los afectados ${ }^{18}$.

Los cuestionarios de valoración de limitaciones y calidad de vida son de uso común en traumatología y reumatología para dolor lumbar. Nuestros resultados utilizando el test de Oswestry confirman la relación entre las limitaciones que implica la lumbalgia en los días de baja de la persona afectada. Como limitación del estudio a la hora de valorar esta asociación reseñar que, al tratarse de un estudio transversal no podemos asegurar la causalidad temporal. Sin embargo consideramos que estos datos abren una puerta a la investigación para que estudios prospectivos posteriores confirmen esta asociación.

En la valoración del paciente con dolor lumbar es importante medir su repercusión funcional. Las pruebas complementarias, de laboratorio y de imagen no informan sobre la situación clínica del paciente ni sobre los cambios que se producen. Las escalas de valoración son consideradas como otras pruebas complementarias que, aunque menos utilizadas, aportan información sobre la intensidad del dolor y su repercusión en las actividades de la vida cotidiana con el sesgo que implica la subjetividad de los cuestionarios, aunque tienen el aspecto positivo de que son fáciles de emplear e interpretar como herramienta de apoyo. La escala de incapacidad por dolor lumbar de Oswestry es, junto con la escala de Roland-Morris ${ }^{19}$, la más utilizada y recomendada a nivel mundial. Su utilización permitirá al clínico obtener información desde la perspectiva del paciente, conocer el grado de eficacia de las distintas técnicas de tratamiento empleadas y comparar resultados con otros estudios publicados en la literatura.

\section{CONCLUSIÓN}

Dadas las implicaciones que el dolor lumbar supone desde un punto de vista sanitario y social, las actuaciones preventivas y terapéuticas deberán tener en consideración aspectos sociodemográficos básicos como la edad, sexo del paciente, nivel cultural y su entorno, y apoyarse en cuestionarios de valoración, de los que el de Oswestry, utilizado en este trabajo, junto con la escala de Roland-Morris son los más recomendados en la literatura científica para relacionar limitaciones y discapacidad/incapacidad.

Agradecimientos: Al Hospital Clínico Universitario de Valencia y a los pacientes procedentes de la Unidad de Traumatología Clínico-Malvarrosa que se incluyeron en el estudio.

Dolor Lumbar en pacientes de consulta traumatológica. Variables socio-sanitarias relacionadas Ma Teófila Vicente-Herrero, Servio Tulio Casal-Fuentes, Gemma Victoria Espíl-López, Alejandro Fernández-Montero 


\section{REFERENCIAS BIBLIOGRÁFICAS}

1. Edwards J, Hayden J, Asbridge M, Gregoire B, Magee K. Prevalence of low back pain in emergency settings: a systematic review and meta-analysis. MC Musculoskelet Disord. 2017 Apr 4;18(1):143.

2. Hoy D, Brooks P, Blyth F, Buchbinder R. The Epidemiology of low back pain. Best Pract Res Clin Rheumatol. 2010 Dec;24(6):769-81.

3. Vlaeyen JWS, Maher CG, Wiech K, Van Zundert J, Meloto CB, Diatchenko L, et al. Low back pain. Nat Rev Dis Primers. 2018 Dec 13;4(1):52.

4. Patrick N, Emanski E, Knaub MA. Acute and chronic low back pain. Med Clin North Am. 2014 Jul;98(4):77789, xii.

5. Cuantificación del consumo de alcohol. Fisterra. Ayuda en consulta; 2013. Disponible en: https://www fisterra.com/ayuda-en-consulta/calculos/cuantificacion-consumo-alcohol/ (Consultado el 9 de enero de 2018).

6. Serrano-Atero MS, Caballero J, Cañas A, García-Sau - ra PL, Serrano-Álvarez C, Prieto J. Pain assess - ment (I). Rev Soc Esp Dolor 2002; 9: 94-108.

7. Flórez García MT, García Pérez MA, García Pérez F, Armenteros Pedreros J, Álvarez Prado A, Martínez Lorente MD. Adaptación transcultural a la población española de la escala de incapacidad por dolor lumbar de Oswestry. Rehabilitación (Madr). 1995;29:138-45.

8. Alcántara Bumbiedro S, Flórez García MT, Echávarri Pérez C, García Pérez F. Oswestry low back pain disability questionnaire. Rehabilitación (Madr). 2006;40(3):150-8.

9. Selva Sevilla C, Ferrara P, Gerónimo Pardo M. Psychometric Properties Study of the Oswestry Disability Index in a Spanish Population with Previous Lumbar Disc Surgery: Homogeneity and Validity. Spine (Phila Pa 1976). 2018 Sep 17.

10. Sheahan PJ, Nelson-Wong EJ, Fischer SL. A review of culturally adapted versions of the Oswestry Disability Index: the adaptation process, construct validity, test-retest reliability and internal consistency. Disabil Rehabil. 2015;37(25):2367-74.

11. Chiarotto A, Maxwell LJ, Terwee CB, Wells GA, Tugwell P, Ostelo RW. Roland-Morris Disability Questionnaire and Oswestry Disability Index: Which Has Better Measurement Properties for Measuring Physical Functioning in Nonspecific Low Back Pain? Systematic Review and Meta-Analysis. Phys Ther. 2016 Oct;96(10):1620-37.

12. Smeets R, Köke A, Lin CW, Ferreira M, Demoulin C. Measures of function in low back pain/disorders: Low Back Pain Rating Scale (LBPRS), Oswestry Disability Index (ODI), Progressive Isoinertial LiftingEvaluation (PILE), Quebec Back Pain Disability Scale (QBPDS), and Roland-Morris Disability Questionnaire (RDQ). Arthritis Care Res (Hoboken). 2011 Nov;63 Suppl 11:S158-73.

13. Assessment of Patient-Reported Outcome Instruments to Assess Chronic Low Back Pain. Ramasamy A, Martin ML, Blum SI, Liedgens H, Argoff C, Freynhagen R, et al. Assessment of Patient-Reported Outcome Instruments to Assess Chronic Low Back Pain.Pain Med. 2017 Jun 1;18(6):1098-1110.

14. Werneke M, Hayes D, Deutscher D. Clinical utility of the Oswestry Disability Index for measuring the function of patients with low back pain. Spine J. 2018 Apr;18(4):712-713.

15. Ohtori S, Ito T, Yamashita M, Murata Y, Morinaga T, Hirayama J, et al. Evaluation of low back pain using the Japanese Orthopaedic Association BackPain Evaluation Questionnaire for lumbar spinal disease in a multicenter study: differences in scores based on age, sex, and type of disease. J Orthop Sci. 2010 Jan;15(1):86-91.

16. Fehrmann E, Kotulla S, Fischer L, Kienbacher T, Tuechler K, Mair P. et al. The impact of age and gender on the ICF-based assessment of chronic low back pain. Disabil Rehabil. 2018 Jan 12:1-10.

17. Billis E, Koutsojannis C, Matzaroglou C, Gliatis J, Fousekis K, Gioftsos G et al. Association of low back pain on physical, sociodemographic and lifestyle factors across a general population sample within Greece. J Back Musculoskelet Rehabil. 2017;30(2):279-290.

18. Arshad R, Pan F, Reitmaier S, Schmidt H. Effect of age and sex on lumbar lordosis and the range of motion. A systematic review and meta-analysis. J Biomech. 2019 Jan 3;82:1-19.

19. Roland M, Fairbank J. The Roland-Morris Disability Questionnaire and the Oswestry Disability Questionnaire. Spine (Phila Pa 1976). 2000 Dec 15;25(24):3115-24. 


\section{ANEXO}

Test de evaluación funcional de Oswestry

\begin{tabular}{lc}
\hline \multicolumn{1}{c}{ ÍTEM } & PUNTUACIÓN \\
\hline 1. Intensidad del dolor: & \\
- Actualmente no tengo dolor ni de columna ni de pierna & $\square 0$ \\
- Mi dolor de columna o pierna es muy leve en este momento & $\square 1$ \\
- Mi dolor de columna o pierna es moderado en este momento & $\square 2$ \\
- Mi dolor de columna o pierna es intenso en este momento & $\square 3$ \\
- Mi dolor de columna o pierna es muy intenso en este momento & $\square 4$ \\
- Mi dolor es el peor imaginable en este momento & $\square 5$ \\
\hline 2. Actividades de la vida cotidiana (lavarse, vestirse, etc.): & \\
- Las hago sin ningún dolor & $\square 0$ \\
- Puedo hacer de todo solo y en forma normal, pero con dolor & $\square 1$ \\
- Las hago en forma más lenta y cuidadosa por el dolor & $\square 2$ \\
- Ocasionalmente requiero ayuda & $\square 3$ \\
- Requiero ayuda a diario & $\square 4$ \\
- Necesito ayuda para todo, estoy en cama & $\square 5$ \\
\hline 3. Levantar objetos: & $\square 0$ \\
- Puedo levantar objetos pesados desde el suelo sin dolor & $\square 1$ \\
- Puedo levantar objetos pesados desde el suelo pero con dolor & $\square 2$ \\
- No puedo levantar objetos pesados desde el suelo debido al dolor, pero & \\
- si cargar un objeto pesado desde una mayor altura, ej. desde una mesa & $\square 3$ \\
- Solo puedo levantar desde el suelo objetos de peso mediano & $\square 4$ \\
- Solo puedo levantar desde el suelo cosas muy ligeras & $\square 5$ \\
- No puedo levantar ni cargar nada & $\square 0$ \\
\hline 4. Caminar: & $\square 1$ \\
- Camino todo lo que quiero sin dolor & $\square 2$ \\
- No puedo caminar más de 1-2 km debido al dolor & $\square 5$ \\
- No puedo caminar más de 500-1000 m debido al dolor & $\square$ puedo caminar apoyado por uno o dos bastones \\
\hline - Senácticamente en cama, me cuesta mucho hasta ir al baño & $\square$ \\
\hline
\end{tabular}

5. Sentarse:

- Me puedo sentar en cualquier silla todo el rato que quiera sin dolor $\quad \square 0$

- Solo en un asiento especial puedo sentarme sin dolor

- No puedo estar sentado más de una hora sin dolor

- No puedo estar sentado más de 30 minutos sin dolor

- No puedo estar sentado más de 10 minutos sin dolor

- No puedo permanecer ningún instante sentado sin que sienta dolor

6. Pararse:

- Puedo permanecer de pie lo que quiero sin dolor

- Puedo permanecer de pie lo que quiero, pero con dolor

- No puedo estar más de una hora parado libre de dolor

- No puedo estar parado más de 30 minutos sin dolor

- No puedo estar parado más de 10 minutos sin dolor

- No puedo permanecer ningún instante de pie sin dolor

7. Dormir:

- Puedo dormir bien, libre de dolor

- Ocasionalmente el dolor me altera el sueño

- Por el dolor no logro dormir más de 6 horas seguidas

- Por el dolor no logro dormir más de 4 horas seguidas

- Por el dolor no logro dormir más de 2 horas seguidas 
8. Actividad sexual:

- Normal, sin dolor de columna

- Normal, aunque con dolor ocasional de columna

- Casi normal, pero con importante dolor de columna

- Seriamente limitada por el dolor de columna

- Casi sin actividad por el dolor de columna

- Sin actividad, debido a los dolores de columna

9. Actividades sociales (fiestas, deportes, etc.):

- Sin restricciones, libres de dolor

- Mi actividad es normal, pero aumenta el dolor

- Mi dolor tiene poco impacto en mi actividad social, excepto aquellas más enérgicas (ej. deportes)

- Debido al dolor salgo muy poco

- Debido al dolor no salgo nunca

- No hago nada debido al dolor

10. Viajar:

- Sin problemas, libre de dolor

- Sin problemas, pero me produce dolor

- El dolor es severo, pero logro viajes de hasta 2 horas

- Puedo viajar menos de 1 hora por el dolor

- Puedo viajar menos de 30 minutos por el dolor

- Solo viajo para ir al médico o al hospital

Modificado de: Flórez García MT, et al. Adaptación transcultural a la población española de la escala de incapacidad por dolor lumbar de Oswestry. Rehabilitación (Madr). 1995;29:138-45. 